\title{
The Effect of Degrading Binocular Single Vision on Fine Visuomotor Skill Task Performance
}

\author{
Marianne E. F. Piano, ${ }^{1}$ and Anna R. O'Connor ${ }^{2}$ \\ ${ }^{1}$ Department of Vision Sciences, Glasgow Caledonian University, Lanarkshire, United Kingdom \\ ${ }^{2}$ Directorate of Orthoptics and Vision Sciences, University of Liverpool, Liverpool, United Kingdom
}

Correspondence: Anna R. O'Connor, University of Liverpool, Thompson Yates Building, Brownlow Hill, Liverpool L69 3GB;

annaoc@liv.ac.uk.

Submitted: September 11, 2012 Accepted: October 28, 2013

Citation: Piano MEF, O'Connor AR. The effect of degrading binocular single vision on fine visuomotor skill task performance. Invest Ophthalmol Vis Sci. 2013;54:8204-8213. DOI: $10.1167 /$ iovs.12-10934
Purpose. To evaluate the impact of degrading binocular single vision (BSV) on performance of fine visuomotor skill tasks requiring speed/accuracy.

Methods. Binocular functions (Frisby/Preschool Randot [PSR] stereoacuity, horizontal phasic prism fusion amplitudes) were measured in visually normal participants aged 18 to 40 years $(n=80)$. Participants performed 2 -timed visuomotor tasks: water pouring $(450 \mathrm{~mL}$ accurately into five measuring cylinders at $90 \mathrm{~mL}$ ) and bead threading on upright needles (30 large, 22 small beads, creating two difficulty levels). Task and binocular function measures were repeated in a randomized order with monocular visual acuity (VA) reduced in three-line increments using convex spherical lenses. Analyses used were Kruskal-Wallis/Mann-Whitney $U$ tests and linear mixed modeling.

Results. Median Frisby stereoacuity levels were $20^{\prime \prime}$ arc at baseline, $55^{\prime \prime}$ arc when VA was degraded by 6 lines, 210" arc by 9 lines, and unmeasurable by 12 lines ( 9 lines in some individuals). Task performance times deteriorated for the large bead task ( $7 \%-10 \%$ between lenses, total $37 \%$ from median baseline time of 51 seconds, $P<0.001$ ), and small bead task (0.5\%-15\% between lenses, total $42 \%$ from median baseline time of 57 seconds, $P<0.001$ ). Binocular function measures causing significant fixed effects were base-out fusional amplitudes in both bead tasks (large: $P=0.010$, small: $P=0.011$ ) and PSR stereoacuity in the small bead task $(P=0.047)$. Water-pouring task performance was not significantly affected by changes in any experimental parameter.

Conclusions. Degrading motor fusion as well as stereoacuity significantly affects performance in certain fine visuomotor tasks. This impact is differentially affected by task difficulty.

Keywords: binocular vision, fine motor tasks, motor fusion, stereopsis
$B^{i}$ inocular single vision (BSV) is the ability to use both eyes together to achieve a single fused percept, even in the presence of disparity of the image seen by each eye. It is divided into five grades: simultaneous perception, superimposition, sensory fusion, motor fusion, and stereopsis. Subjectively, individuals who have lost their BSV have reported a significant impact on their daily life. ${ }^{1-3}$ Studies have attempted to quantify the extent and nature of the contribution BSV makes to aspects of visuomotor function, such as judging depth, ${ }^{4-7}$ visually guided reaching/grasping kinematics ${ }^{7-9}$ and trajectories, ${ }^{10}$ negotiating obstacles, ${ }^{11}$ and time taken to complete motor tasks requiring spatial awareness/certainty and manual dexterity. 5,6,12-15

Focusing on the relationship between BSV and the ability to perform fine visuomotor tasks, many of these studies ${ }^{4-6,13,14}$ used subjects with normal BSV and compared performance under binocular versus monocular viewing conditions. Only some studies compared those with normal BSV to those with a long-term absence of $\mathrm{BSV},{ }^{4-6,9,13}$ or additionally included participants with reduced BSV. ${ }^{5,6,9,12,15}$ In two of these papers, ${ }^{5,6}$ subjects with absent and reduced BSV were collapsed together for analysis due to insufficient numbers in the groups. Thus, it appears the main focus is on the difference between having BSV and not having BSV, and the contribution made by reduced $\mathrm{BSV}$ has been evaluated in less detail. In addition, in the majority of studies looking at reduced BSV, the only grade focused upon is stereoacuity. Only O'Connor et al. ${ }^{5}$ attempted to establish whether deficits in motor fusion (the BSV grade preceding stereoacuity) also affected fine visuomotor task performance. The number of individuals with reduced motor fusion in their study group was limited $(n=7)$, but findings indicated that the motor fusion deficits were associated with poorer visuomotor task performance when compared with individuals with normal motor fusion.

Study findings comparing individuals with a long-term absence of BSV to individuals temporarily deprived of BSV have also been shown to conflict. Those with a long-term absence of BSV have been shown to perform better, 5,6 equivalent to, ${ }^{13}$ or worse than ${ }^{4,9}$ individuals temporarily deprived of normal BSV. The task batteries and viewing conditions differed in these studies, accounting for some variability in the findings. However, the conflicting findings may be due to variable adaptation between individuals to losses in binocular function, or indeed individual differences in ability to perform the study task batteries, independent of binocular status.

Individuals with reduced/absent BSV recruited for the above studies also had amblyopia in many cases, where the deficit in vision extends beyond a simple reduction in clarity: Distortions of stimulus orientation and position can occur during 
amblyopic eye viewing. ${ }^{16}$ Individuals with strabismic and/or anisometropic amblyopia (or history thereof) also struggle with end-stage object grasping, ${ }^{8,9,17}$ planning, ${ }^{7,10,18}$ and execution $^{7-10,17,18}$ of visually guided reaching, and completion of manual dexterity tasks requiring speed and/or accuracy along with visual input. ${ }^{5,6,12,15}$ Thus the inclusion of subjects with amblyopia within the samples could also potentially affect the results.

It is difficult to differentiate between amblyopia, degraded stereoacuity, the presence of strabismus, or a combination of these, as the cause of slower performance in motor tasks. One study has examined this and concluded that strabismus appeared to be the only contributing factor. ${ }^{15}$ However, in our previous study, ${ }^{6}$ only 29 of 143 subjects had a constant deviation, and a significant difference in performance was still found between the binocular and nonbinocular groups. Another study ${ }^{12}$ also found task performance to be affected in preschool children with unilateral vision impairment and no strabismus. In the absence of strabismus, stereoacuity was found to be the main predictor for performance on neurodevelopmental tasks requiring visuomotor integration, fine handeye coordination, and visuospatial skills. All three studies ${ }^{6,12,15}$ appear to agree that the level of monocular visual acuity (VA) is not a major contributor to effects upon task performance. This is supported by a study ${ }^{19}$ where mild anisometropic amblyopia was associated with poorer visually guided reaching performance than that of controls without amblyopia, who were monocularly blurred to the same mean acuity level $(0.400$ $\log$ MAR). Reducing VA to that level in the control group failed to equalize task performance between the two groups.

However, studies evaluating the impact of monocular and binocular blur upon performance of visually normal individuals in motor tasks such as car steering, ${ }^{20}$ obstacle negotiation, ${ }^{21,22}$ visually guided reaching ${ }^{19}$ or grasping, ${ }^{23}$ and certain sports, ${ }^{24-26}$ have found that the effects of experimentally induced image blur can also be task dependent. It is possible that in cases where VA is degraded due to amblyopia, the impact of this will be task-dependent also. Therefore, the choice of task, presence of strabismus, and level of binocular function (as well as the neural deficits associated with amblyopia) could all contribute, in combination, to the degradations in visuomotor task performance, for individuals with amblyopia.

The current study aimed to examine the contribution that motor fusion and stereoacuity make to visuomotor task performance, while addressing some of these confounding factors. Individual differences in task performance and adaptation to BSV deficits were minimized using a repeated measures design, in which participants with normal BSV and no amblyopia or strabismus had their fine visuomotor task performance assessed as their BSV was progressively degraded.

\section{MeTHODS}

\section{Subjects}

Subjects with normal stereoacuity and good vision in both eyes were recruited from the University of Liverpool and Glasgow Caledonian University. The inclusion criteria were age 18 to 40 years, VA $\leq 0.30 \log$ MAR in each eye, interocular acuity difference $\leq 0.200 \operatorname{logMAR}$, near point of convergence $<10$ $\mathrm{cm},{ }^{27}$ stereoacuity $\leq 60$ " arc measured with the Preschool Randot (PSR; Stereo Optical Co., Inc., Chicago, IL) stereotest, no known ophthalmic defect (other than refractive error), and no known physical impairment affecting motor skills. The meeting of the last two criteria was determined via case history. Informed written consent was obtained before participation. This research protocol was approved by the University of Liverpool and Glasgow Caledonian University Research Ethics Committees, and follows the tenets of the Declaration of Helsinki.

\section{Ophthalmic Measures}

Monocular and binocular VA, assessed with the participant's up-to-date refractive correction (not more than 2 years old), was evaluated using a Bailey-Lovie letter logMAR chart (HaagStreit UK, Harlow, UK) at $3 \mathrm{~m}$. All subjects had a binocular VA of 0.125 logMAR or better. The chart was changed between eyes to reduce letter memorization. Threshold acuity was scored by letter at the point where three letters or more on a line were identified incorrectly after two attempts.

Baseline measurements of binocular functions were also taken. Convergence was assessed three times using a Royal Air Force (RAF) rule (Haag-Streit UK) to obtain an average. A 4diopter (D) prism reflex test was performed at $33 \mathrm{~cm}$ using a foveal fixation target, to ensure central fixation was present in both eyes. Horizontal phasic prism fusion range (a measure of motor fusion) was recorded at $33 \mathrm{~cm}$ and $6 \mathrm{~m}$ using a target letter that was sized two lines above threshold acuity, with blur, break, and recovery points recorded for prism base in (BI) and then base out (BO) ranges. These measure divergent and convergent fusional reserves, respectively. This provided a measurement of residual fusional reserves after any latent deviation had been controlled. Stereoacuity was measured using the PSR stereotest at $40 \mathrm{~cm}$, and the Frisby stereotest (Frisby Stereotest, Sheffield, UK). A real-depth stereotest (Frisby) was used in addition to the PSR stereotest because real depth tests are more resistant to the effects of monocular blur. ${ }^{28}$

The Frisby stereotest plate (I, II, or III) was mounted on a frame to hold it at a $90^{\circ}$ angle to the table surface, against a white background. A chinrest was used to reduce head movement and thus minimize monocular cues. Assessment was performed using a four-alternative forced-choice staircase procedure adapted from Costa et al. ${ }^{29}$ Our viewing distance started at $50 \mathrm{~cm}$ with plate I and pass criterion was two out of three presentations correct, with viewing distance changed by $10-\mathrm{cm}$ increments/decrements. Threshold was recorded as ceiling (passing plate III at $80 \mathrm{~cm}$ ), unmeasurable (unable to detect plate I at $30 \mathrm{~cm}$ ), or best stereoacuity achievable after three reversals.

Participants viewed reference pictures for the PSR test during the baseline condition only, to avoid memorization of the picture locations. Threshold was determined by pass criterion of two of three pictures correctly named and located.

\section{Degrading Binocular Functions}

A protocol was then followed to produce decremental changes in binocular functions. The eye with the poorest corrected distance VA, or the nondominant eye in cases where VA was equal (determined using the Porta test), ${ }^{30}$ was selected. This eye was then progressively blurred using convex spherical lenses to achieve 6-, 9-, and 12-line reductions in monocular VA. The lens power required to achieve these reductions was recorded. These decrements were chosen based on data from a subset of the sample $(n=18 / 73)$ indicating that a 3-line reduction in VA produced little change in binocular functions or motor task performance. A recent publication ${ }^{19}$ found a 3line reduction in VA in visually normal subjects via monocular blur did not influence visually guided reaching kinematics. Although the paper does not mention whether binocular function was also unchanged by this, reaching is an important 
part of the tasks used in our study. Therefore, these findings support our choice of VA decrements.

The data for the subset at baseline, 6- and 9-line VA reduction, and at suppression, was included in the analysis. However, subjects in the subset were not entered into the additional 12-line monocular VA reduction condition as they were tested in the 3-line condition instead. Of those participating in the 12-line condition $(n=55), 14$ experienced a breakdown in binocular vision during this condition and therefore did not complete it. This condition was excluded from analysis for these individuals. Subsequently, sample sizes for the baseline, 6- and 9-line VA reduction, and suppression lens conditions were $n=73$, and the 12-line VA reduction lens condition was $n=41$.

Lastly, lens power was increased to produce total monocular suppression, using Bagolini glasses as a control. Where diplopia persisted and the participant was unable to suppress, lens power was increased until the second image could be comfortably ignored. This created four lens conditions: three in which increasing amounts of anisometropia were induced to degrade BSV, and one in which BSV was no longer measurable. If a breakdown in binocular vision (e.g., manifest strabismus with suppression or diplopia on Bagolini glasses) occurred with any lens prior to the one inducing suppression, no subsequent lens conditions were required.

\section{Fine Visuomotor Skills Tasks}

Participants performed two tasks to assess fine visuomotor skill function. These were a water-pouring task $(450 \mathrm{~mL}$ of water into five measuring cylinders in fixed positions, quickly and accurately up to the 90-mL mark, marked with white tape), and a bead-threading task with two difficulty levels. One level was large bead-threading $(30 \times 20$-mm black beads onto $2 \times 5$-mm knitting needles in fixed position at participant's midline, with the needles horizontally separated by $11.5 \mathrm{~cm}$ ). The other level was small bead-threading $(22 \times 10$-mm black beads onto 2 -mm knitting needle in fixed position at participant's midline). The visuomotor task battery was selected from two previous papers. ${ }^{5,6}$

A bead-threading task was selected due to the requirement for visually guided precision grasping to pick up the bead, and accurate reaching in order to place the bead on the needle. Disparity has been shown to play a part in grip formation and reach movement times in normal subjects, ${ }^{23,31}$ and in individuals with poor binocular function and amblyopia, visually guided reaching and grasping have both been shown to be affected. ${ }^{7-10,17,18}$ Our previous studies ${ }^{5,6}$ showed that task completion time in stereo-deficient and normal participants was longer when bead/needle size was reduced, so we included large and small bead/needle sizes to assess task difficulty effects. It has been suggested that individuals with amblyopia and poor binocular function struggle with online corrections of visually guided reaching errors, due to using degraded visual feedback. ${ }^{9}$ We therefore selected the waterpouring task as it requires rapid integration of visual feedback (the rising water level) for online modifications of pouring speed, and depth judgments to aid spatial localization of receptacle position.

In the current study, participants performed the visuomotor tasks twice at baseline (normal binocular function, no lenses in place) to minimize practice effects. Only the second attempt was used for analysis. In the individual lens conditions, visuomotor tasks were performed once, to minimize the impact of fatigue effects from the multiple lens conditions. All tasks were performed using the subject's preferred hand, and the positions of the water jug and bead trays were moved to be closest to this hand. Participants were only allowed to use one hand throughout the tasks and were not allowed to touch the needle in the bead-threading task. Performance times were measured with a stopwatch, rounding up from milliseconds to the nearest second to account for stopwatch button presses. Accuracy on the waterpouring task was taken by reading from the bottom of the meniscus at eye level, recording in milliliters. Participants were instructed to complete each task "as quickly and accurately as possible," to ensure that they selected their own speed/accuracy tradeoff for each task. Following the baseline condition, visuomotor tasks and binocular function measures (motor fusion, Frisby/PSR stereoacuities) were repeated once for each of the four lens conditions (6- to 12-line reduction in VA and suppression). Task performance order and lens conditions were randomized to minimize practice and fatigue effects.

In total, the ophthalmic examination took 20 minutes to complete, and the task battery 40 minutes. Lens condition randomization was achieved by assigning integers to tasks and lens conditions in turn, and randomizing the order using the RAND function in spreadsheet software (Microsoft Excel; Microsoft Corp., Redmond, WA).

\section{Statistical Analysis}

Statistical analyses were performed using statistical software (SPSS 17; SPSS, Inc., Chicago, IL). The independent variable was VA, altered by fixed amounts as a result of the variable power hypermetropic spherical lenses applied. Dependent variables were motor fusion, Frisby/PSR stereoacuity, and task performance measures. Task performance measures were water-pouring accuracy/performance time, and large/small bead-threading performance time. For the task performance measures, water-pouring accuracy amounts were converted to absolute values to reduce the impact of data skew from extreme under-pours due to water spillage. Stereoacuity values were converted to $\log$ units for analysis, with a value of 4.0 being ascribed to cases where stereoacuity was not measurable with the stereotests.

Descriptive statistics were used to define baseline characteristics of the subjects, with parametric statistics used for continuous measures (age and distance VA), and nonparametric statistics for noncontinuous measures or skewed data (task performance measures, motor fusion, and Frisby/PSR stereoacuity). A Spearman's rank correlation was also performed post hoc, to evaluate the tradeoff between accuracy and performance time in the water-pouring task.

All statistical analyses for changes in the dependent variables were performed on the difference between the lens condition and baseline, with comparisons made between lens conditions (i.e., baseline versus 3-line VA reduction, compared with the baseline versus 6-line VA reduction, etc.), and to baseline (i.e., baseline versus 3-line VA reduction, compared with zero change). This approach was adopted to reduce the impact of individual differences in time taken to complete the visuomotor tasks, and decrements in binocular function in response to monocular blur.

Kruskal-Wallis tests were used to identify whether there were significant differences in motor performance task time and binocular functions (prism fusion break and recovery range, and stereoacuity), when the independent variable (VA) was changed. A $P$ value of $\leq 0.05$ was required to achieve statistical significance. If a significant difference was found, individual Mann-Whitney $U$ tests were performed post hoc to identify the lenses at which a significant change in performance or binocular function occurred, when compared with baseline. A Mann-Whitney $U$ test was also 
TABLE 1. Baseline Characteristics of Participants Recruited to the Study

\begin{tabular}{lc}
\hline \multicolumn{1}{c}{ Baseline Characteristics } & Participants, $\boldsymbol{n}=73$ \\
\hline Sex, $n$ & 44 female, 29 male \\
Age, y, mean \pm SD & $24.43 \pm 4.91$ \\
Best-corrected distance & $-0.10 \pm 0.15$ \\
Binocular VA, logMAR, mean \pm SD & \\
Mean spherical equivalent refractive error, DS, mean \pm SD \\
OD & $-1.21 \pm 2.55$ \\
OS & $-1.15 \pm 3.00$
\end{tabular}

Near horizontal phasic prism fusion amplitude, $\Delta$

BI, median (IQR) [min, max] $\begin{array}{ll}\text { Break } & 16(4)[6,25] \\ \text { Recovery } & 12(4)[4,20]\end{array}$

BO (IQR) [min, max]

Break $30(15)[10,45]$

Recovery 25 (15) $[8,45]$

Frisby stereoacuity, log "arc, median (IQR) [min, max]

Preschool Randot stereoacuity, log "arc, median (IQR) [min, max]

$1.48(0.30)[1.30,1.78]$

Convergence and 4-D prism reflex results were normal in all participants.

performed to compare performance times between the two bead-threading tasks at baseline, to evaluate the effect of the smaller beads and thinner needle (increased task difficulty) on task performance. A Bonferroni correction was applied to the post hoc Mann-Whitney $U$ tests (one for each lens condition, four in total). A $P$ value of $\leq 0.0125$ was therefore required to achieve statistical significance for these tests.

\section{Linear Mixed Modeling Analysis}

Linear mixed modeling (LMM) was performed to estimate the relationship between task performance times (dependent variable), and the covariates of VA and binocular function (Frisby/PSR stereoacuity and BI/BO motor fusion break amplitudes) across the repeated measure of lens condition. Lens power was excluded as a covariate to minimize the occurrence of multicollinearity, as although the correlation between lens power and VA was only significant when VA was reduced by 12 lines (Spearman's rho $=0.559, P<0.001$ ), lens power values were closely tied to visual acuity decrements. The linear mixed model assumes a random intercept for each subject. This is important in a repeated measures design as used in our study because each subject participated in each lens condition. As a result, measures taken within individual lens conditions are not statistically independent. This produces correlated data and correlated errors within subjects and within lens conditions (e.g., if a participant is particularly slower than average at the beadthreading task, they will be in all five experimental conditions). LMM analysis accounts for this by specifying the nature of this correlation (covariance structure), and has the added advantage over conventional repeated measures ANOVA procedures of including subjects with missing data. This is especially important for our study, as a proportion of subjects were not tested in the lens C (12-line VA reduction) condition due to having lost their BSV with that strength of lens.

\section{Constructing the Model}

The individual effect of lens condition and the interactions between lens condition and each covariate (e.g., lens condition $\times \mathrm{VA}$ ) were examined as fixed factors, using a scaled identity covariance structure. This structure specifies that variance in each lens condition is constant and covariance between lens conditions is independent. Individual task performance measures were log-transformed to normalize their distribution for LMM analysis. Water accuracy outcome measures were not suitable for log transformation due to many zero values. Based on its nonsignificance in the nonparametric analysis for all lens conditions, this was omitted from LMM as a dependent variable. Lens condition $\mathrm{D}$ was also omitted from the model due to many of its values reverting to a fixed value (e.g., stereoacuity becoming 4.00, motor fusion becoming 0 ). Likelihood ratios were used to compare model fit with a baseline model that had no covariates, and previous model versions. Likelihood ratio was calculated as the difference of the $-2 \log$ likelihood (-2LL) measure of the two models, and compared to a $\chi^{2}$ distribution to obtain a measure of statistical significance. The -2LL measures how likely the experimental data is to occur with the estimated contributions of the covariates (parameter estimates). The model that significantly differed from the basic model and had the lowest $-2 \mathrm{LL}$ value was selected as the model of choice. A $P$ value of $\leq 0.05$ was required to achieve statistical significance when comparing with the $\chi^{2}$ distribution, and for hypothesis testing of parameters in the LMM.

Covariates included in the model were also evaluated for collinearity using the variance inflation factor (VIF). A high VIF value $(>10)$ indicates that a covariate is linearly dependent on one or more other covariates. This signifies the covariate has inflated variance, which may adversely affect the parameter estimates coming out of the model. All covariates in our model had a VIF $<3$ and were permissible to be included within the model.

\section{Exclusion of Outliers}

For performance times in each task, and for binocular functions, scatter plots of the difference between baseline and each lens condition were conducted prior to modeling. This was performed to identify outliers that would substantially affect model fit. Two subjects were excluded from the dataset for all models (one with a $>100$-second difference between baseline and lens $\mathrm{B} / \mathrm{C}$ for both bead-threading tasks, and one who lost binocular vision at lens $\mathrm{B}$ and only completed three lens conditions).

Residuals for the water-pouring task were evaluated and found to be homoscedastic and normally distributed with no prominent outliers. Residuals for the large bead task were not normally distributed by Shapiro-Wilk test (large beads, $W=0.871, P<0.001)$, as well as containing some prominent positive and negative outliers limiting homoscedascity. Residuals for the small bead task were normally distributed but contained prominent negative outliers limiting homoscedascity. These outliers were removed (large beads task, $n=5$, standardized residuals $>0.1$; small beads task, $n=2$, standardized residuals $>0.1$ ) and the selected model was repeated, creating residuals that were homoscedastic and normally distributed. The final sample sizes for the model were $n=71$ for the water-pouring task ( $n=40$ for lens C); $n=66$ for the large bead task $(n=37$ for lens C); and $n=69$ for the small bead task ( $n=40$ for lens C). 
TABLE 2. Impact of Hypermetropic Spherical Lens Power on Monocular Visual Acuity in the Eye Viewing Through the Lens, by Lens Used

\begin{tabular}{lccccc}
\hline \multicolumn{1}{c}{ Binocular Test } & Baseline & Lens A & Lens B & Lens C & Lens D, Suppression \\
\hline $\begin{array}{l}\text { Lens power, DS, mean } \pm \text { SD } \\
\begin{array}{l}\text { Visual acuity in eye wearing lens, } \\
\text { logMAR, mean } \pm \text { SD }\end{array}\end{array}$ & $+0.00 \pm 0.00$ & $+1.36 \pm 0.26^{*}$ & $+2.27 \pm 0.51^{*}$ & $+3.14 \pm 0.55^{*}$ & $+6.18 \pm 2.54^{*}$ \\
& $\begin{array}{c}\text { OD: }-0.10 \pm 0.14 \\
\text { OS: }-0.10 \pm 0.15\end{array}$ & $0.50 \pm 0.14^{*}$ & $0.81 \pm 0.13^{*}$ & $1.11 \pm 0.13^{*}$ & $1.58 \pm 0.10^{*}$ \\
\hline
\end{tabular}

Monocular visual acuity decreases in at least 0.30 increments as lens power increases, except for lens D. A higher lens power was required to induce suppression, producing more substantial reduction in monocular visual acuity.

${ }^{*}$ Change in lens power/visual acuity between this condition and the baseline condition is significant $(P<0.0125$ using Mann-Whitney $U$ test, Bonferroni correction).

\section{Results}

Seventy-three subjects were recruited to the study, with $95 \%$ aged $<35$ years. Their baseline characteristics are shown in Table 1. Convergence and 4-D prism reflex results were normal in all participants (convergence near point $<10 \mathrm{~cm},{ }^{27} 4-\mathrm{D}$ prism reflex movement demonstrated in each eye). All participants successfully completed the study. The convex spherical lenses required to produce sufficient blur are shown in Table 2.

\section{Effect of Monocular Retinal Defocus and Reduced VA on Binocular Functions}

$\mathrm{BI}$ and BO prism fusion amplitudes and stereoacuity changed with increasing defocus and stepwise reduction of monocular VA (Table 3, Kruskal-Wallis test results at $\leq 0.05$ significance level denoted by *). Table 3 shows reducing prism fusion break/recovery amplitudes and stereoacuity as lens power increases and VA reduces. Mann-Whitney $U$ tests indicated these reductions were significant compared with baseline.

PSR stereotest responses degraded more as VA reduced, compared with the Frisby stereotest. Of the 34 individuals with no measurable PSR stereoacuity when monocular VA was degraded by 12 lines (sample size for this condition $n=41$ ), 23 (68\%) had no measurable Frisby stereoacuity. The reduction in stereoacuity with increasing anisometropia was more marked than that of prism fusion break/recovery amplitudes. Table 3 shows that the median prism fusion BO break amplitudes only dropped below normal limits (defined as $\geq 25 \Delta \mathrm{BO}$ and $\geq 10$ $\Delta \mathrm{BI})^{30}$ when monocular VA was reduced by nine lines or more, while median BI break amplitudes remained within normal limits. Figure 1 shows BI and BO prism fusion break amplitude against PSR stereoacuity for baseline and Lens C (12-line reduction in monocular VA). For many subjects, BI prism fusion break amplitudes remained within normal limits at lens C (upper half of the graph) while PSR stereoacuity dropped below normal limits (defined as $\leq 1.78 \mathrm{log}$ "arc), ${ }^{32,33}$ into the right-hand side of the graph. $\mathrm{BO}$ prism fusion break amplitudes drop below normal limits for more subjects at lens $\mathrm{C}$.

\section{Effect of Reduced Binocular Functions on Visuomotor Task Performance}

The small bead-threading task took significantly longer to complete than the large bead-threading task at baseline, despite there being only 22 beads to be threaded rather than 30 (median difference $=6$ seconds, interquartile range $[\mathrm{IQR}]=$ 6, $U=1295.5, P<0.001, r=0.63$ ). This shows that there was an effect of task difficulty on task performance times. When evaluating water-pouring task performance times against accuracy, no significant correlations were identified.

As BSV measures declined (Table 3), changes in task performance for all three visuomotor tasks occurred (Table 4, Kruskal-Wallis test results at 0.05 significance level denoted by *). Table 4 shows small changes in performance times (per individual cylinder) and absolute accuracy for the waterpouring task, which were not linearly related to changes in

TABLE 3. Impact of Declining VA on Binocular Function (Phasic Horizontal Prism Fusion Break/Recovery Amplitudes, Stereoacuity) by Lens Used

\begin{tabular}{|c|c|c|c|c|c|c|c|c|c|}
\hline \multirow[b]{2}{*}{ Binocular Test } & \multicolumn{2}{|c|}{ Baseline } & \multicolumn{2}{|c|}{ Lens $\mathrm{A}$} & \multicolumn{2}{|c|}{ Lens $B$} & \multicolumn{2}{|c|}{ Lens $\mathrm{C}$} & \multirow{2}{*}{$\begin{array}{c}\text { Lens D, } \\
\text { Suppression }\end{array}$} \\
\hline & BI & BO & BI & BO & BI & BO & BI & BO & \\
\hline \multicolumn{10}{|c|}{ Prism fusion amplitude, break point, $\Delta^{*}$} \\
\hline $\begin{array}{l}\text { Median (IQR) } \\
\text { [Min, Max] }\end{array}$ & $\begin{array}{c}16(5.5) \\
{[6,25]}\end{array}$ & $\begin{array}{c}32.5(15) \\
{[10,45]}\end{array}$ & $\begin{array}{l}15(6) \\
{[6,25]}\end{array}$ & $\begin{array}{l}25(19) \\
{[8,45] \dagger}\end{array}$ & $\begin{array}{l}14(5.5) \\
{[4,25] \dagger}\end{array}$ & $\begin{array}{c}20(16) \\
{[6,45]+\ddagger}\end{array}$ & $\begin{array}{c}12(8) \\
{[4,20] \dagger}\end{array}$ & $\begin{array}{c}16(15) \\
{[1,45] \dagger \neq}\end{array}$ & $\begin{array}{c}0(0) \\
{[0,0] \dagger \neq}\end{array}$ \\
\hline \multicolumn{10}{|c|}{ Prism fusion amplitude, recovery point, $\Delta^{*}$} \\
\hline $\begin{array}{l}\text { Median (IQR) } \\
\text { [Min, max] }\end{array}$ & $\begin{array}{l}12(6) \\
{[4,20]}\end{array}$ & $\begin{array}{l}25(15) \\
{[8,45]}\end{array}$ & $\begin{array}{l}12(6) \\
{[2,20]}\end{array}$ & $\begin{array}{l}20(12.5) \\
{[4,45] \dagger \ddagger}\end{array}$ & $\begin{array}{c}12(6) \\
{[2,20] \dagger}\end{array}$ & $\begin{array}{c}18(15) \\
{[2,45] \dagger \ddagger}\end{array}$ & $\begin{array}{c}8(8) \\
{[1,18] \dagger \neq}\end{array}$ & $\begin{array}{c}12(14) \\
{[0,45] \dagger \ddagger}\end{array}$ & $\begin{array}{c}0(0) \\
{[0,0]+\ddagger}\end{array}$ \\
\hline \multicolumn{10}{|l|}{ Frisby, $\log$ "arc* } \\
\hline $\begin{array}{l}\text { Median (IQR) } \\
\text { [Min, max] }\end{array}$ & \multicolumn{2}{|c|}{$\begin{array}{c}1.30(0.00) \\
{[1.30,1.60]}\end{array}$} & \multicolumn{2}{|c|}{$\begin{array}{c}1.74(0.53) \\
{[1.30,2.53] \dagger}\end{array}$} & \multicolumn{2}{|c|}{$\begin{array}{c}2.33(0.74) \\
{[1.74,4.00] \dagger}\end{array}$} & \multicolumn{2}{|c|}{$\begin{array}{c}4.00(1.42) \\
{[1.88,4.00]+\delta}\end{array}$} & $\begin{array}{c}4.00(0.00) \\
{[4.00,4.00]+\S}\end{array}$ \\
\hline $\begin{array}{l}\text { Preschool Randot, log "arc" } \\
\text { Median (IQR) } \\
\text { [Min, max] }\end{array}$ & $\begin{array}{l}1.48 \\
{[1.30}\end{array}$ & $\begin{array}{c}(0.30) \\
1.78]\end{array}$ & $\begin{array}{r}2.00 \\
{[1.48}\end{array}$ & $\begin{array}{l}(0.65) \\
4.00]+\varsigma\end{array}$ & $\begin{array}{r}2.90 \\
{[1.78,}\end{array}$ & $\begin{array}{l}(1.70) \\
4.00]+\S\end{array}$ & $\begin{array}{r}4.00 \\
{[2.30}\end{array}$ & $\begin{array}{l}(0.00) \\
4.00]+\$\end{array}$ & $\begin{array}{c}4.00(0.00) \\
{[4.00,4.00]+\delta}\end{array}$ \\
\hline
\end{tabular}


BI Break Amplitude vs. PSR Stereoacuity

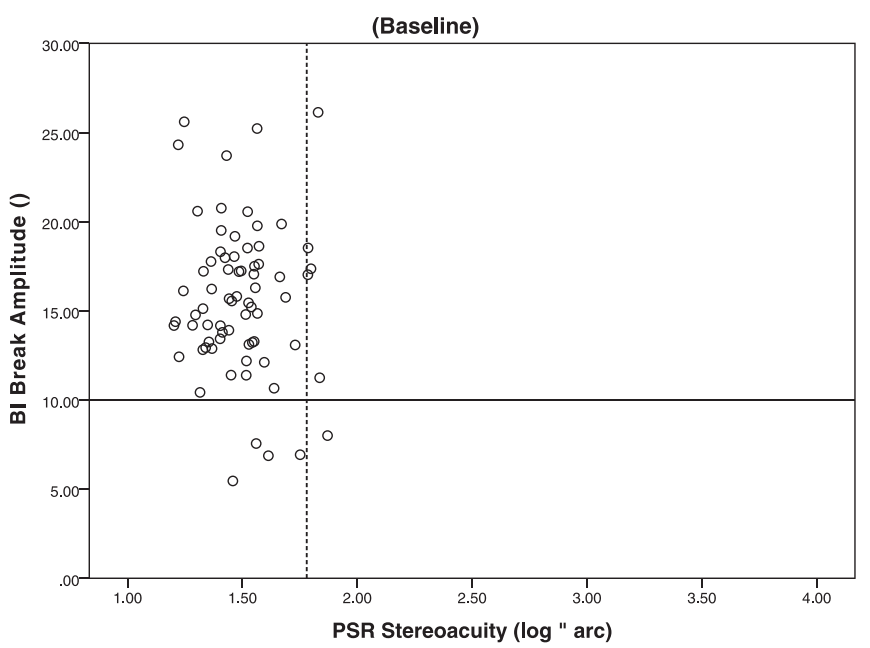

BO Break Amplitude vs. PSR Stereoacuity

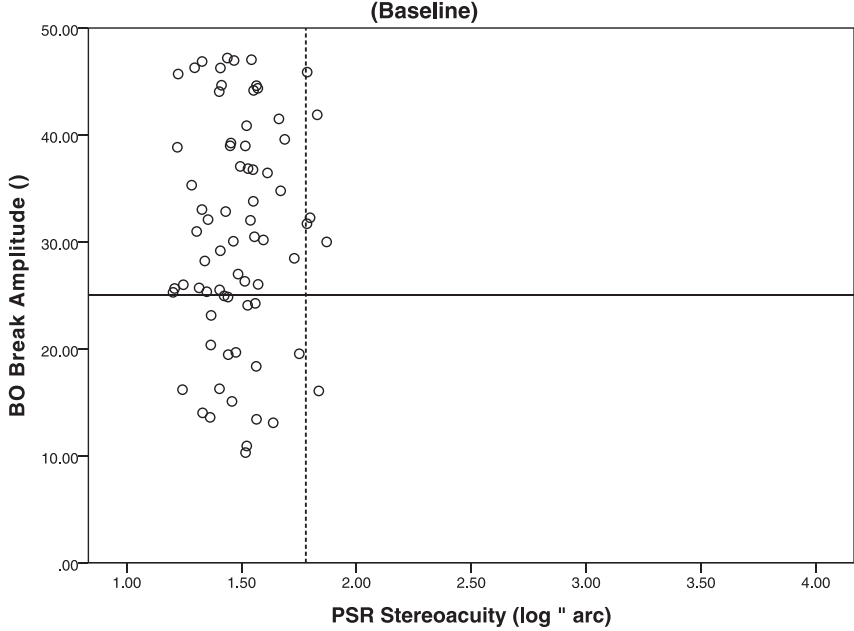

BI Break Amplitude vs. PSR Stereoacuity

(Lens C)

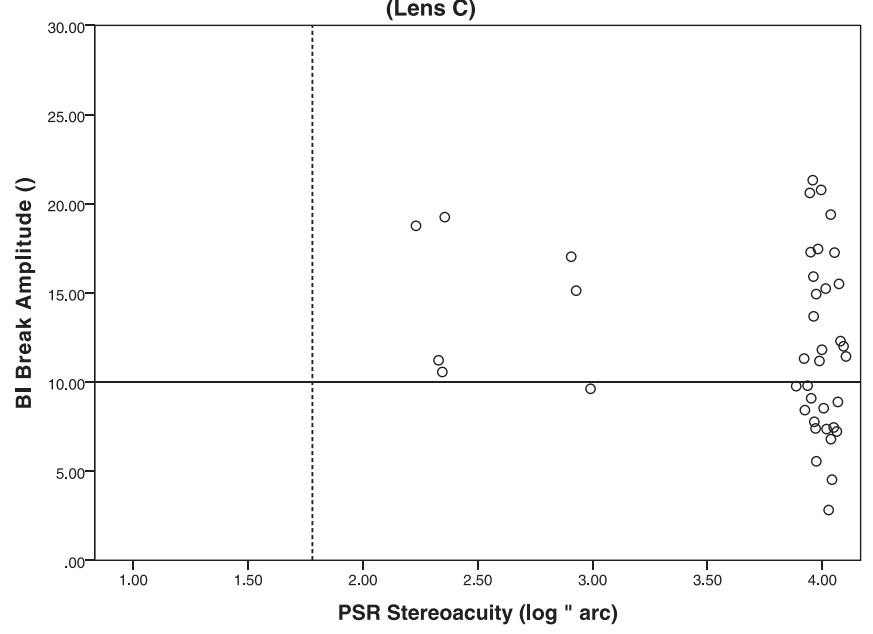

BO Break Amplitude vs. PSR Stereoacuity

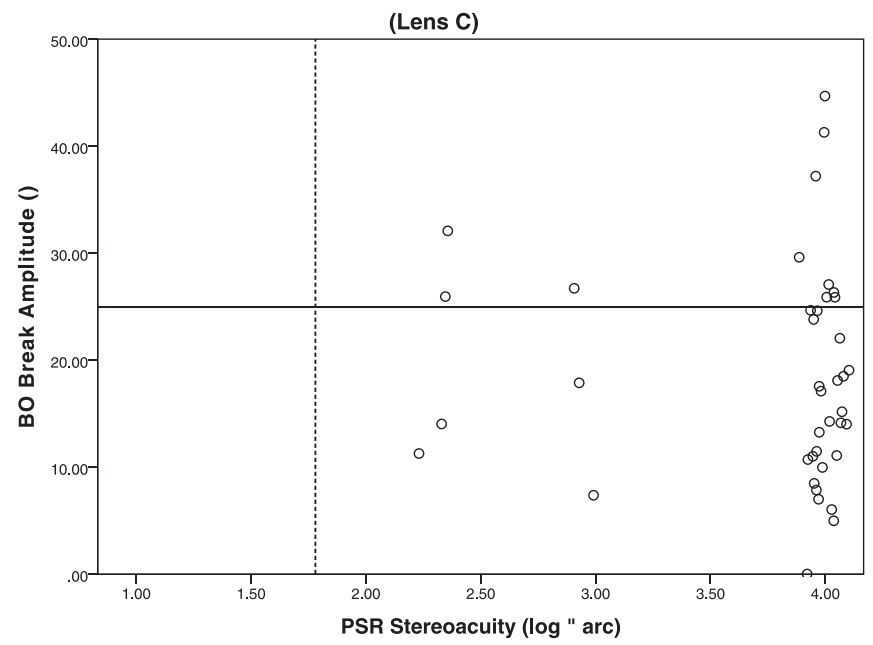

Figure 1. Changes in horizontal phasic prism fusion BI (top) and BO (bottom) break amplitudes against PSR stereoacuity at baseline (left, $n=80$ ), compared with lens $\mathrm{C}$ (right, $n=43$ ). The intersecting lines indicate the normative values for horizontal phasic prism fusion break amplitude (borizontal line) and PSR stereoacuity (vertical line).

BSV. In some cases, changes reached statistical significance when compared with baseline using Mann-Whitney $U$ tests (Table 4). However, the total median change (per cylinder) from baseline to the suppression condition was 1 second for time taken $(\mathrm{IQR}=1, \mathrm{U}=1971, P=0.004, r=0.34$, and $0 \mathrm{~mL}$ for accuracy $[\mathrm{IQR}=2])$, which was not statistically significant.
For the bead-threading tasks a larger change in performance occurred as BSV was degraded (Table 4). A median change from baseline to the final lens condition of 19 seconds occurred for the large bead-threading task (37\% increase in time, $\mathrm{IQR}=14, \mathrm{U}=0, P<0.001, r=1.31)$ and 24 seconds for the small bead-threading task ( $42 \%$ increase in time, IQR $=19$,

TABLE 4. Impact of Declining Binocular Function on Water-Pouring Task Time and Accuracy, by Lens Used

\begin{tabular}{cccccc}
\hline \multicolumn{1}{c}{ Task } & Baseline & Lens A & Lens B & Lens C & $\begin{array}{c}\text { Lens D, } \\
\text { Suppression }\end{array}$ \\
\hline $\begin{array}{c}\text { Water-pouring time, s/cylinder, } \\
\text { median (IQR) }[\text { min, max }]\end{array}$ & $5(2)[3,9]$ & $5(1)[3,8]$ & $5(2)[3,8]$ & $5(2)[3,10]$ & $6(1)[3,8] \dagger$ \\
$\begin{array}{c}\text { Absolute water-pouring accuracy, } \\
\text { mL, }{ }^{*} \text { median (IQR) }[\text { min, max }]\end{array}$ & $0(1)[-3,3]$ & $0(2)[-4,5]$ & $0(1)[-3,3]$ & $0(2)[-4,4]$ & $0(2)[-4,7]$ \\
$\begin{array}{c}\text { Large beads, s, } \\
\quad \text { median (IQR) }[\text { min, max }]\end{array}$ & $51(9)[32,63]$ & $53(9)[46,69] \dagger$ & $59(9)[43,165] \dagger$ & $65(17)[42,93] \dagger$ & $70(15)[48,108] \dagger$ \\
$\begin{array}{c}\text { Small beads, s, } \\
\text { median }(\mathrm{IQR})[\min , \max ]\end{array}$ & $57(13)[46,78]$ & $61(12)[46,80] \dagger$ & $65(13)[47,163] \dagger$ & $72(20)[43,130] \dagger$ & $81(16)[52,111] \dagger$
\end{tabular}

* Statistically significant change in performance in response to reduction in monocular VA $(P<0.05$ using Kruskal-Wallis test).

† Difference in performance between this condition and the baseline condition is significant $(P<0.0125$ using Mann-Whitney $U$ test, Bonferroni correction). 


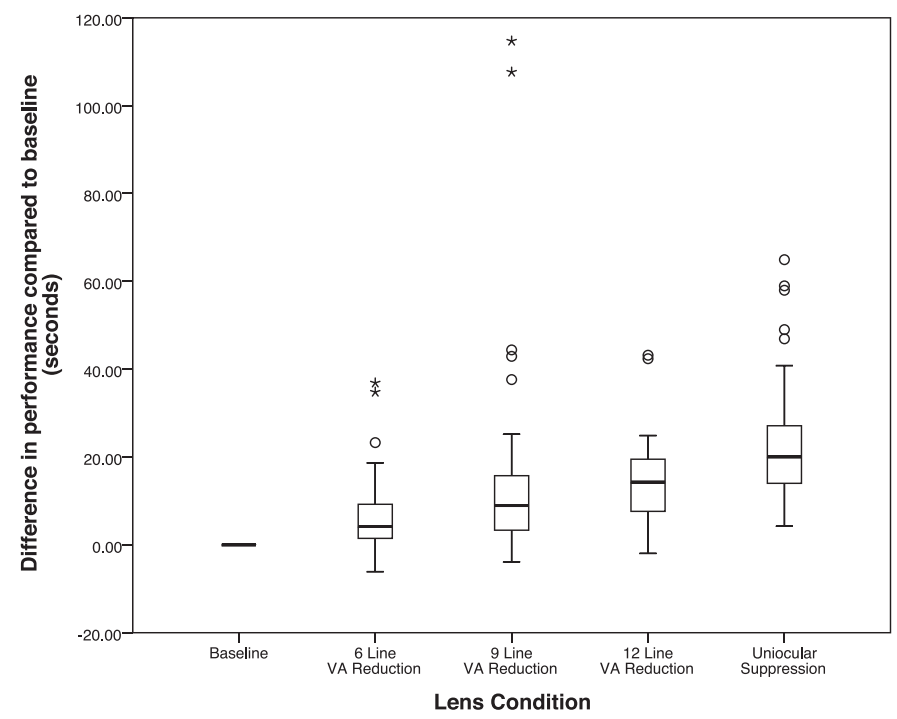

Figure 2. Effect of degrading binocular function on large bead-threading time, by lens condition, when compared with baseline. Box represents interquartile range with median dividing the upper and lower quartiles. Error bars represent data falling $1.5 \times \mathrm{IQR}$ above and below the 25 th and 75th percentiles. Circles indicate outliers within 1.5 to $3 \times \mathrm{IQR}$ range, while asterisks indicate outliers $>3 \times \mathrm{IQR}$.

$\mathrm{U}=73, P<0.001, r=1.27)$. The pattern of change in performance times for the large (Fig. 2) and small (Fig. 3) beadthreading tasks shows that degradation of task performance times increases with BSV degradation, in a nonlinear pattern. The longer task completion times associated with increased task difficulty are preserved as BSV degrades (see also Table 4). Task performance degradation was most marked when BSV was no longer measurable with clinical tests.

During linear mixed modeling analysis, the model that was significantly different from the base model and possessing the lowest $-2 \log$ likelihood contained covariates of $\mathrm{BI}$ and $\mathrm{BO}$ break amplitudes, Frisby and PSR stereoacuity, and VA (-2LL for large beads $=-621.72$, small beads $=-566.26$, waterpouring $=-324.48)$. Correlations between the two stereotest results were strong when lenses were applied (e.g., lens A Spearman's rho $=0.493, P<0.001$ ), but both stereotests had a fusion break amplitudes also had a low VIF $(<2)$, despite having a Spearman's rho of $0.420(P<0.001)$ in the lens A condition. Correlations between motor fusion break amplitudes and stereotest results were weak (Spearman's rho < -0.35 ), with fluctuating statistical significance across lens conditions, but again it was possible to include them in the same model due to the low VIF values.

In the water-pouring task, there were no statistically significant fixed effects. In the large bead-threading task, there was a significant fixed effect of $\mathrm{BO}$ break amplitude $\left(\mathrm{F}_{4,164}=\right.$ $2.551, P=0.041$ ) by lens condition. Parameter estimates for the two bead-threading tasks are shown in Supplementary Table S1. For the large bead-threading task, when lenses were applied, increasing BO break amplitudes were associated with a static unit decrease in performance time across lens conditions (lens A to $\mathrm{C}=-1.00$ seconds), which was statistically significant for lens C $(95 \% \mathrm{CI}=-1.01,-1.00, t=$

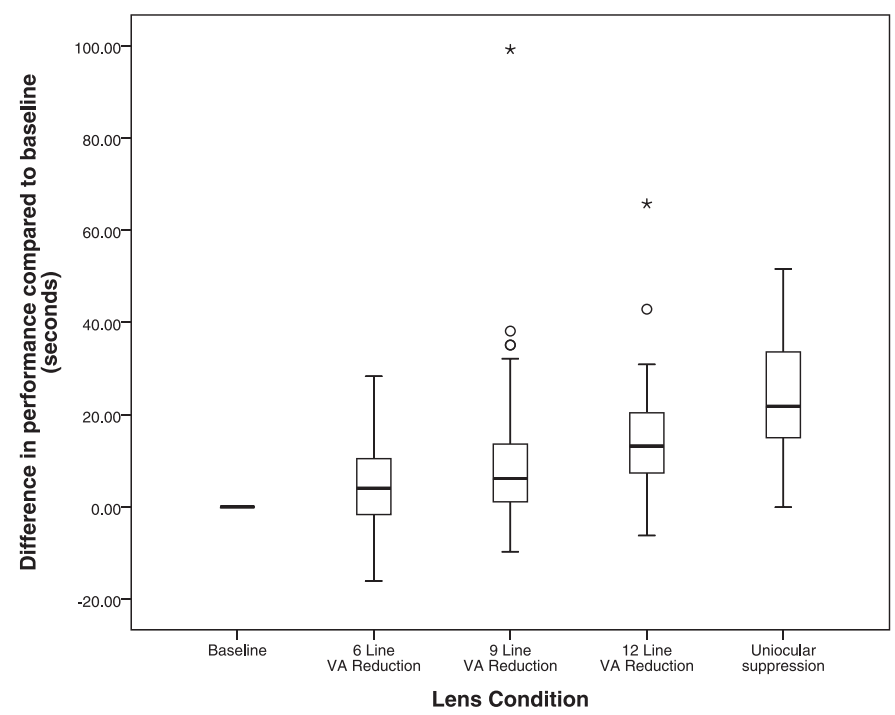

FiguRE 3. Effect of degrading binocular function on small bead-threading time, by lens condition, when compared with baseline. Box represents interquartile range with median dividing the upper and lower quartiles. Error bars represent data falling $1.5 \times \mathrm{IQR}$ above and below the 25 th and 75th percentiles. Circles indicate outliers within 1.5 to $3 \times \mathrm{IQR}$ range, while asterisks indicate outliers $>3 \times \mathrm{IQR}$. 
$-2.45, P=0.015)$. This can be interpreted as an increase in performance time as $\mathrm{BO}$ break amplitudes worsen.

In the small bead-threading task, there were significant fixed effects of $\mathrm{BO}$ break amplitude $\left(\mathrm{F}_{4,180}=3.057, P=0.018\right)$ and PSR stereoacuity $\left(\mathrm{F}_{4,178}=2.692, P=0.033\right)$. Parameter estimates (Supplementary Table S1) showed that when lenses were applied, increasing BO break amplitudes were associated with a similar static unit decrease in performance times as the large bead-threading task (lens $\mathrm{A}$ and $\mathrm{B}=-1.00$ second, lens $\mathrm{C}$ $=-1.01$ seconds), again interpreted as increasing performance times with worsening BO break amplitudes. This was statistically significant for lens B $(95 \% \mathrm{CI}=-1.01,-1.00, t=$ $-2.77, P=0.006)$ and lens $\mathrm{C}(95 \% \mathrm{CI}=-1.01,-1.00, t=-2.35$, $P=0.020)$. An exponential unit increase in performance times occurred with worsening PSR stereoacuity, and this was statistically significant at lens B $(+1.04$ seconds, $95 \% \mathrm{CI}=$ $1.00,1.07, t=2.01, P=0.046)$.

\section{Discussion}

In this study, we used monocular blur to reduce VA, in a group of participants with normal BSV. The subsequent degradation of their level of BSV was associated with a significant increase in time taken to complete the two bead-threading tasks. LMM analysis indicated that degrading binocular functions contributed to this change in a way that was differentially affected by task difficulty. Water-pouring performance time and accuracy were not affected in a way that could be considered clinically relevant, even in the suppression lens condition. Changes in performance time for this task were also unaffected by VA or binocular functions in LMM analysis, suggesting it can be performed adequately with monocular visual information alone. No significant correlation between performance time and accuracy was found for this task, indicating that the standardized instructions were successful in producing an equal speed/accuracy trade-off for this task.

Findings from our nonparametric analysis for the waterpouring task are in common with our previous studies. ${ }^{5,6}$ Existing research demonstrates that people with normal BSV have poorer performance on certain visuomotor tasks when completing them monocularly, as opposed to binocularly. ${ }^{4-6,13,14}$ Our finding of increased bead-threading task performance times in the suppression lens conditions corroborates this research. However, our study also shows that performance in such a task is affected when BSV is reduced. It is known that these effects occur in individuals with reduced or absent BSV who have amblyopia/strabismus, ${ }^{4-6,9,12,13,15}$ but the current study demonstrates that this can occur with degraded BSV, independent of the presence of strabismus/ amblyopia.

A substantial amount of monocular blur was required to generate abnormal levels of binocular function that affected performance on our visuomotor tasks. Real depth stereoacuity, as measured with the Frisby stereotest, only became unmeasurable when lenses of +3.00 spherical diopters (DS) or greater (on average, see Tables 2,3) were applied. The finding of more degraded PSR stereoacuity with increasing monocular blur, in comparison to Frisby stereoacuity, also corroborates existing research. ${ }^{28}$ In addition to stereoacuity, we found motor fusion also appears resistant to monocular blur in certain individuals. Median BO break/recovery amplitudes only dropped below normal levels $(\geq 25 \Delta \mathrm{BO})^{34}$ when monocular VA was reduced by nine lines or more. Meanwhile, BI break amplitudes remained within normal limits $(\geq 10 \Delta \mathrm{BI}),{ }^{34}$ even with a 12 line reduction in monocular VA. It appeared that convergent fusional reserves (BO break amplitudes) were more affected by our paradigm than divergent reserves (BI break amplitudes).
While many studies ${ }^{20,24-26}$ have examined the effect of blur on hand-based motor tasks, the defocus is often the same in each eye. However, it has been shown that stereoacuity is more affected when there is a disparity in the amount of retinal defocus between eyes, compared with when it is equal. ${ }^{35}$ Only a few studies $8,19,23$ have examined the relationship between monocular retinal defocus and hand-based visuomotor tasks, with reported effects varying by degree of defocus and task type. Monocular retinal defocus appears to have an impact on movement time and grip formation during reach-to-grasp actions in three-dimensional (3-D) space, with defocus levels of +2.00 to $3.75 \mathrm{DS}^{23}$ However, a study evaluating reaching kinematics in a 2-D reach-to-point task, with defocus levels of +0.75 to 2.00 DS, found reach movement time was not significantly affected. ${ }^{19}$ Grasping was not examined in the 2-D reach-to-point study, so it is not known whether this is similarly unaffected by lower levels of defocus. However, another study ${ }^{8}$ found a higher incidence of grasping errors after object contact with higher levels of defocus (+3.50-5.00 DS), compared with lower levels $(+2.00-3.50$ DS). This would suggest that, in general, larger amounts of defocus cause more of an impact on such kinematics during performance of certain visuomotor tasks. Although we did not measure reach-to-grasp kinematics in our study, the increase in bead-threading task completion times with increasing amounts of defocus is likely attributable to these effects. There is greater reliance in the bead-threading tasks on accurate reaching and precision grasping compared with the water-pouring task, and even more demand for this in the small bead-threading task.

Although the two levels of defocus used in the previously described study ${ }^{8}$ caused changes in measured stereopsis and motor fusion, the direct contribution of these binocular functions to changes in the kinematic parameters was not evaluated. In our study, we attempted to ascertain the contributions made to changes in task performance by the changes in VA and binocular functions. VA decrements were not found to be a significant contributor to performance on any task. In our study, participants were viewing binocularly throughout the task, and were therefore able to utilize visual input from the eye that was not wearing any lenses. Under these circumstances, it is not surprising that task performance was not significantly affected by the VA decrements, despite this being the primary manipulation performed. The findings tie in with those of studies in amblyopic subjects, which have not found reduced $\mathrm{VA}$ in the amblyopic eye to interact significantly with binocular visuomotor task performance. $6,12,15$

Despite over half the participants in the 12-line VA reduction condition having no measurable stereoacuity on either clinical test ( 23 of 41 ), degradations in bead-threading task performance are largest in the suppression lens condition, where motor fusion and stereoacuity are removed. Task performance also continues to deteriorate between the 12-line VA reduction condition (lens $\mathrm{C}$ ) and the suppression lens condition (lens D), rather than plateauing in line with the unmeasurable stereoacuity. Given these two findings, there is a strong argument that motor fusion (still present in all participants in the former lens condition) plays a role in this decline. This is supported by our LMM analysis, in which degraded BO fusional break amplitudes was identified as a significant contributor to increases in performance times for both bead-threading tasks, in the 12-line VA reduction condition (Supplementary Table S1). It was also a significant contributor in the nine-line VA reduction condition (lens B) for the small bead-threading task. The contributory role of motor fusion to performance times in visuomotor tasks such as those used in our study is not one identified in the literature previously. 
Interestingly, declining stereoacuity was not found to be a significant contributor to increasing performance times in the large bead-threading task. This task requires the same reaching and grasping movements as the small bead-threading task, but less accuracy and precision in these actions due to using larger objects. Although we cannot account for the contributions to task performance made by coarser levels of stereoacuity $\left(>800^{\prime \prime}\right.$ arc), or stereoacuity types not measured by our test battery, such as dynamic stereopsis, it would seem that reliance on disparity cues is less for this lower difficulty version of the task. Therefore, the impact of degraded disparity feedback upon performance times for this task is less. However, it has been suggested that online correction of visually guided reaching and grasping is limited in circumstances where access to disparity information is the least, ${ }^{9}$ and in our task that requires these motor skills, it is possible that the absence of disparity cues affected performance on this task in a way that degraded disparity cues did not.

With an increase in task difficulty (small beads task), degraded stereoacuity becomes a significant contributor to increases in performance time, with larger increases per unit degradation of stereoacuity as monocular blur increases and measured stereoacuity worsens. This was statistically significant for lens B only. The lack of statistical significance at lens C is associated with wider confidence intervals, as only seven individuals in the lens $C$ condition had a PSR stereoacuity value that was not 4.00. As the largest parameter estimate for this variable occurs in the lens $\mathrm{C}$ condition, it is a fair assumption that degraded PSR stereoacuity in this condition would have been a contributor of statistical significance were it not for this factor. Although effects are small (complete loss of PSR stereoacuity is associated with a 3 -second increase in performance time), the results demonstrate greater reliance on disparity cues where more reach accuracy and grasp precision is required. As mentioned above, degraded BO break amplitudes were a significant contributor to an increase in task performance times, and when task difficulty is increased, reliance on motor fusion for good task performance occurs with lower levels of blur (9-line VA reduction condition). A 10 $\Delta$ loss of BO break amplitude equated to a 10 -second increase in performance times. Overall, it can be said that increasing the difficulty of the bead-threading task causes greater reliance on disparity and motor fusion cues, which in turn begins to affect performance at a lower level of blur. The greater impact of degraded binocular functions upon task performance when task demands are increased is another finding not identified previously in the literature.

For the water-pouring task, LMM analysis results seemed to indicate that adequate performance on this task can be achieved using monocular visual information alone. A possible reason for this is the primary visuomotor demands for the water-pouring task are planning and execution of the reaching movement to position the jug for pouring. These actions are based on disparity/vergence judgments of cylinder position, as well as utilizing visual feedback from the rising water level to decide when to terminate pouring. The changing water fill line (or lack thereof in spillages) provides continuous information midtask that facilitates rapid corrections of reaching shortfalls/ overshoots, in comparison with the bead-threading tasks where errors are only correctable by repeating the grasp or hand movement. For example, mis-grasps cause the bead to be missed on the plate and require repetition of the grasp, and errors in reaching cause collision with the needle or shortfalls/ overshoots, leading to dropping of the bead or the need to repeat the action at a different reach distance. Therefore, the relative ease of correcting errors in the water-pouring task may have mitigated the impact of degraded binocular visual feedback upon task performance, in comparison with tasks requiring precision grasping movements, such as the beadthreading tasks.

Another factor potentially influencing the impact of degraded binocular visual feedback upon performance in any of our tasks is the fact that free head movement was allowed on all tasks to create naturalistic viewing conditions, allowing use of monocular cues to improve performance. However, the impact of degraded BSV on bead-threading task performance under these conditions indicates that use of monocular cues does not completely compensate for the deficit in certain motor tasks. Monocular cues have also been found to compensate inadequately for reaching and grasping deficits in amblyopic subjects who have stereoacuity losses. ${ }^{9}$

Our LMM analysis has provided some insight into the way in which binocular functions contributed to performance on the visuomotor tasks used in this study. However, it is important to highlight one caveat pertaining to the construction of our model. Figure 1 demonstrates that fusional amplitudes were relatively independent from stereoacuity measures in this study. Also, collinearity diagnostics indicated that it was possible to include both measures of stereoacuity as well as BI and BO break amplitudes in our chosen model, without a high VIF affecting results. However, it should be borne in mind that there is a theoretical dependence upon motor fusion for the existence of stereopsis as we know it clinically. Although we cannot guarantee that model fit has not been influenced by this dependency, the best fit (smallest -2LL score) was with all of these covariates included. In addition, it is known that a limited amount of disparity information can be extracted from diplopic images, ${ }^{36}$ and even within our sample there were individuals who possessed motor fusion reserves below normal limits, but still had normal stereopsis on PSR stereotesting. This would suggest that the dependency is not complete.

Overall, the primary unique finding of this study is inhibited performance on the visuomotor task of bead-threading when normal BSV is reduced. Findings of existing studies demonstrating degraded visuomotor task performance in the absence of BSV have also been corroborated. Our other study findings include evidence that:

1. Motor fusion contributes to normal visuomotor task performance regardless of task difficulty;

2. Increased task difficulty facilitates more reliance on vergence and disparity cues, causing stereoacuity to become a significant contributing factor to performance; and

3. The impact on task performance times caused by degraded binocular functions is compounded by increased task difficulty.

Our study highlights the role of motor fusion and stereopsis in normal performance on the visuomotor tasks evaluated. With the role of decorrelated binocular experience in amblyogenesis being given increased prominence in a recent amblyopia review, ${ }^{37}$ our findings suggest that it is important to improve all aspects of BSV as part of any treatment regime to improve binocular function.

\section{Acknowledgments}

The authors thank Anita Simmers for her support during the data collection phase at Glasgow Caledonian University, Gabriela Czanner for statistical advice, and Stephanie Rossit for advice on use of reaching and grasping terminology.

Supported by a Fight for Sight PhD Studentship during the data collection phase at Glasgow Caledonian University (MP).

Disclosure: M.E.F. Piano, None; A.R. O'Connor, None 


\section{References}

1. Brady FB. A Singular View. 3rd ed. Annapolis: Brady; 1985.

2. Godber G. Living with one eye. BMJ. 1987;295:1351.

3. Romano PE. A case of acute loss of binocular vision and stereoscopic depth perception. (The misery of acute monovision, having been binocular for 68 years). Binocul Vis Strabismus Q. 2003;18:51-55.

4. Brown KC, Buckley D. Do we really need binocular single vision? Br Ir Orthopt J. 2004;1:46.

5. O'Connor AR, Birch EE, Anderson S, Draper H. Relationship between binocular vision, visual acuity, and fine motor skills. Optom Vis Sci. 2010;87:942-947.

6. O'Connor AR, Birch EE, Anderson S, Draper H; FSOS Research Group. The functional significance of stereopsis. Invest Ophthalmol Vis Sci. 2010;51:2019-2023.

7. Niechwiej-Szwedo E, Goltz HC, Chandrakumar M, Hirji Z, Crawford JD, Wong AMF. Effects of anisometropic amblyopia on visuomotor behavior, part 2: visually guided reaching. Invest Ophthalmol Vis Sci. 2011;52:795-803.

8. Melmoth DR, Finlay AL, Morgan MJ, Grant S. Grasping deficits and adaptations in adults with stereo vision losses. Invest Ophthalmol Vis Sci. 2009;50:3711-3720.

9. Suttle CM, Melmoth DR, Finlay AL, Sloper JJ, Grant S. Eye-hand coordination skills in children with and without amblyopia. Invest Ophthalmol Vis Sci. 2011;52:1851-1864.

10. Niechwiej-Szwedo E, Goltz HC, Chandrakumar M, Wong AMF. The effect of sensory uncertainty due to amblyopia (lazy eye) on the planning and execution of visually-guided 3D reaching movements. PLoS One. 2012;7:e31705.

11. Buckley JG, Panesar GK, MacLellan MJ, Pacey IE, Barrett BT. Changes to control of adaptive gait in individuals with longstanding reduced stereoacuity. Invest Ophthalmol Vis Sci. 2010;51:2487-2495.

12. Hrisos S, Clarke MP, Kelly T, Henderson J, Wright CM. Unilateral visual impairment and neurodevelopmental performance in preschool children. Br J Ophthalmol. 2006;90:836838.

13. Joy $\mathrm{S}$, Davis $\mathrm{H}$, Buckley D. Is stereopsis linked to hand-eye coordination? Br Orthopt J. 2001;58:38-41.

14. Sheedy JE, Bailey IL, Buri M, Bass E. Binocular vs. monocular task performance. Am J Optom Physiol Opt. 1986;63:839846.

15. Webber AL, Wood JM, Gole GA, Brown B. The effect of amblyopia on fine motor skills in children. Invest Ophthalmol Vis Sci. 2008;49:594-603.

16. Barrett BT, Pacey IE, Bradley A, Thibos LN, Morrill P. Nonveridical visual perception in human amblyopia. Invest Ophthalmol Vis Sci. 2003;44:1555-1567.

17. Grant S, Melmoth DR, Morgan MJ, Finlay AL. Prehension deficits in amblyopia. Invest Ophthalmol Vis Sci. 2007;48: 1139-1148.

18. Niechwiej-Szwedo E, Goltz HC, Chandrakumar M, Hirji Z, Wong AMF. Effects of anisometropic amblyopia on visuomotor behavior, III: temporal eye-hand coordination during reaching. Invest Ophthalmol Vis Sci. 2011;52:5853-5861.
19. Niechwiej-Szwedo E, Kennedy SA, Colpa L, Chandrakumar M, Goltz HC, Wong AMF. Effects of induced monocular blur versus anisometropic amblyopia on saccades, reaching, and eye-hand coordination. Invest Ophthalmol Vis Sci. 2012;53: 4354-4362.

20. Owens DA, Tyrrell RA. Effects of luminance, blur, and age on nighttime visual guidance: A test of the selective degradation hypothesis. J Exp Psychol Appl. 1999;5:115-128.

21. Vale A, Buckley JG, Elliot DB. Gait alterations negotiating a raised surface induced by monocular blur. Optom Vis Sci. 2008;85:1128-1134.

22. Vale A, Scally AJ, Buckley JG, Elliot DB. The effects of monocular refractive blur on gait parameters when negotiating a raised surface. Ophthalmic Physiol Opt. 2008;28:135142.

23. Melmoth DR, Storoni M, Todd G, Finlay AL, Grant S. Dissociation between vergence and binocular disparity cues in the control of prehension. Exp Brain Res. 2007;183:283298.

24. Bulson RC, Ciuffreda KJ, Hung GK. The effect of retinal defocus on golf putting. Ophthalmic Physiol Opt. 2008;28: 334-344.

25. Mann DL, Ho NY, De Souza NJ, Watson DR, Taylor SJ. Is optimal vision required for the successful execution of an interceptive task? Hum Mov Sci. 2007;26:343-356.

26. Applegate RA, Applegate RA. Set shot shooting performance and visual acuity in basketball. Optom Vis Sci. 1992;69:765768.

27. von Noorden GK, Campos ECC. Binocular Vision and Ocular Motility: Theory and Management of Strabismus. 6th ed. St. Louis, MO: Mosby; 2001.

28. Odell NV, Hatt SR, Leske DA, Adams WE, Holmes JM. The effect of induced monocular blur on measures of stereoacuity. J AAPOS. 2009;13:136-141.

29. Costa MF, Moreira SMCF, Hamer RD, Ventura DF. Effects of age and optical blur on real depth stereoacuity. Ophthalmic Physiol Opt. 2010;30:660-666.

30. Porac C, Coren S. The dominant eye. Psychol Bull. 1976;83: 880-897.

31. Melmoth DR, Grant S. Advantages of binocular vision for the control of reaching and grasping. Exp Brain Res. 2006;171: 371-388.

32. Ohlsson J, Villarreal G, Abrahamsson M, Cavasoz H, Sjostrom A, Sjostrand J. Screening merits of the Lang II, Frisby, Randot, Titmus, and TNO stereo tests. J AAPOS. 2001;5:316-322.

33. Simons K. A comparison of the Frisby, Random-Dot E, TNO, and Randot circles stereotests in screening and office use. Arch Ophthalmol. 1981;99:446-452.

34. Rowe FR. Fusional vergence measures and their significance in clinical assessment. Strabismus. 2010;18:48-57.

35. Wood IC. Stereopsis with spatially-degraded images. Ophthalmic Physiol Opt. 1983;3:337-340.

36. Wilcox LM, Allison RS. Coarse-fine dichotomies in human stereopsis. Vision Res. 2009;49:2653-2665.

37. Birch EE. Amblyopia and binocular vision. Prog Retin Eye Res. 2013;33:67-84. 